\title{
BOUNDED HARMONIC MAPS ON A CLASS OF MANIFOLDS
}

\author{
CHIUNG-JUE SUNG, LUEN-FAI TAM, AND JIAPING WANG
}

(Communicated by Peter Li )

\begin{abstract}
Without imposing any curvature assumptions, we show that bounded harmonic maps with image contained in a regular geodesic ball share similar behaviour at infinity with the bounded harmonic functions on the domain manifold.
\end{abstract}

In this note, among other things we will prove a Liouville type theorem on harmonic maps. It was first proved by $\mathrm{Yau}[\mathrm{Y}]$ that any positive harmonic function on a complete noncompact manifold with nonnegative Ricci curvature must be constant. Later, Cheng $[\mathrm{Cg}]$ proved that any harmonic map with bounded image from a complete noncompact manifold with nonnegative Ricci curvature into a Cartan-Hadamard manifold must be a constant map. Using a similar method, Choi $[\mathrm{Ch}]$ was able to generalize Cheng's result. He showed that any harmonic map from a complete noncompact manifold with nonnegative Ricci curvature into a complete manifold with sectional curvature bounded from above by $K>0$ is a constant map, provided that the image of the harmonic map lies inside a regular geodesic ball (see Definition 1.3). It was proved by Kendall [Ke] that the result is still true by only assuming the domain manifold supports no nonconstant bounded harmonic functions. If we relax the condition that the manifold has nonnegative Ricci curvature outside a compact set, then the theorem of Yau [Y] is no longer true. In fact, the behavior of bounded and positive harmonic functions on a complete noncompact manifold with nonnegative sectional curvature outside a compact set has been studied thoroughly in [L-T 1]. One of the results in [L-T 1] is that if $M$ has nonnegative sectional curvature outside a compact set, then $M$ has finitely many ends (see §1), and a bounded harmonic function defined near the infinity on an end will be asymptotically constant. There are many kinds of manifolds which satisfy the same property. See the examples in $\S 1$. One of the main results we obtain in this note is that if every bounded harmonic function defined near infinity of an end of a manifold $M$ with respect to some compact set is asymptotically constant, then every bounded harmonic map from $M$ into a regular ball of another manifold is also asymptotically constant at the infinity of each end. There is no curvature assumption on $M$. Moreover, such a map will have finite total energy. We will also

Received by the editors December 16, 1994.

1991 Mathematics Subject Classification. Primary 58E20.

The first author was partially supported by NSC grant\# 830208M194030.

The second author was partially supported by NSF grant \#DMS9300422. 
discuss some properties of bounded harmonic maps from such a manifold into a complete noncompact manifold with nonnegative sectional curvature. We will also prove some existence and uniqueness results on harmonic maps. All manifolds in this paper are assumed to be connected.

We would like to thank Shiu-Yuen Cheng and Peter Li for many valuable discussions. The work was done while the second author was visiting the Chinese University of Hong Kong. He would like to thank Xiao Zhang and Tom Y. H. Wan for their interest in this work.

Let us begin with some definitions.

Definition 1.1. An end $E$ of a manifold $M$ is an unbounded component of the complement of some compact, smooth domain $D$ of $M$. In this case, we say that $E$ is an end corresponding to $D$.

For each $D, M \backslash \bar{D}$ has only finitely many unbounded components since $M$ is a connected manifold. Also the boundary of each component is smooth; in particular, each end has smooth boundary. It is also clear that if $D_{1} \subset D_{2}$ are compact, smooth domains of $M$, then the number of ends corresponding to $D_{1}$ is less than or equal to the number of ends corresponding to $D_{2}$. Hence, we say that $M$ has finitely many ends if there exists $b<\infty$ such that the number of ends corresponding to $D$ is less than or equal to $b$ for any compact, smooth domain $D \subset M$. One also observes that in this case there exist an integer $b_{0}<\infty$ and a compact, smooth domain $D_{0} \subset M$ such that the number of ends corresponding to $D$ is $b_{0}$ for each compact, smooth domain $D$ containing $D_{0}$. We say that $b_{0}$ is the number of ends of $M$. In the following, when $M$ has finitely many ends and we say $E$ is an end of $M$, it is understood that $E$ is an end corresponding to $D_{0}$. If there is no such $b_{0}$, then we say $M$ has infinitely many ends.

Definition 1.2. A Riemannian manifold $M$ with boundary $\partial M$ ( $\partial M$ may be empty) is said to be parabolic if it does not admit any positive Green's function satisfying Neumann boundary condition on $\partial M$. Otherwise, it is said to be non-parabolic. In particular, an end $E$ of $M$ is parabolic if it does not admit any positive Green's function satisfying the Neumann boundary condition on $\partial E$, and is nonparabolic otherwise.

We want to prove the following lemma:

Lemma 1.1. Let $M$ be a complete noncompact manifold without boundary. Let $D$ be a bounded domain in $M$ with smooth boundary, and let $E$ be an end of $M$ with respect to D. Suppose every bounded harmonic function defined at the infinity of $E$ is asymptotically constant near infinity. Let $g_{1}, \ldots, g_{k}$ be any finite set of bounded superharmonic functions defined on $E$, with $\liminf _{x \rightarrow \infty}, x \in E g_{j}(x)=0$, for all $1 \leq j \leq k$, and let $g=\max \left\{g_{1}, \ldots, g_{k}\right\}$. Then

$$
\liminf _{\substack{x \rightarrow \infty \\ x \in E}} g(x)=0 .
$$

Proof. Since $\liminf \operatorname{in}_{x \rightarrow \infty, x \in E} g_{j}(x)=0$ for all $j$, so $\liminf _{x \rightarrow \infty, x \in E} g(x) \geq 0$. Suppose the lemma is not true. Then there is $\epsilon>0$ such that

$$
\liminf _{\substack{x \rightarrow \infty \\ x \in E}} g(x) \geq 2 \epsilon .
$$


Let $o$ be a fixed point in $M$. Then there is $R_{0}$ such that for all $1 \leq j \leq k$, for all $x \in E$ with $r(x) \geq R_{0}$, where $r(x)$ is the geodesic distance from $x$ to $o$, we have

$$
g_{j}(x) \geq-\frac{\epsilon}{2 k},
$$

and

$$
g(x) \geq \epsilon .
$$

By (1.2), (1.3) and the definition of $g$, if $x \in E$ with $r(x) \geq R_{0}$, we have

$$
\begin{aligned}
\sum_{j=1}^{k} g_{j}(x) & \geq \epsilon-(k-1) \cdot \frac{\epsilon}{2 k} \\
& >\frac{\epsilon}{2} .
\end{aligned}
$$

For each $R>R_{0}$, and for each $j$, let $h_{R, j}$ be the harmonic function defined on $E \cap\left(B_{o}(R) \backslash B_{o}\left(R_{0}\right)\right)$, such that $h_{R, j}=g_{j}$ on $E \cap \partial B_{o}(R)$ and $E \cap \partial B_{o}\left(R_{0}\right)$. Since $g_{j}$ is bounded, there exists $R_{i} \rightarrow \infty$ such that $h_{j}=\lim _{i \rightarrow \infty} h_{R_{i}, j}$ exists, for $1 \leq j \leq k$. Since $g_{j}$ is superharmonic and $h_{j}$ is harmonic, $h_{j} \leq g_{j}$. In particular

$$
\begin{aligned}
\liminf _{\substack{x \rightarrow \infty \\
x \in E}} h_{j}(x) & \leq \liminf _{\substack{x \rightarrow \infty \\
x \in E}} g_{j}(x) \\
& =0 .
\end{aligned}
$$

By the assumptions of the lemma, $h_{j}$ is asymptotically constant at infinity of $E$, so

$$
\lim _{\substack{x \rightarrow \infty \\ x \in E}} h_{j}(x)=a_{j}
$$

for some nonpositive constant $a_{j}$. However, by (1.4) and the definition of $h_{R, j}$,

$$
\begin{aligned}
\sum_{j=1}^{k} h_{R_{i}, j} & =\sum_{j=1}^{k} g_{j} \\
& \geq \frac{\epsilon}{2}
\end{aligned}
$$

on $E \cap \partial B_{o}(R)$ and $E \cap \partial B_{o}\left(R_{0}\right)$, for all $i$. Hence $\sum_{j=1}^{k} h_{R_{i}, j} \geq \epsilon / 2$ for all $i$. Let $i \rightarrow \infty$; then we have $\sum_{j=1}^{k} h_{j} \geq \epsilon / 2$. This contradicts (1.5). The proof of the lemma is completed.

Using a similar argument, one can obtain the following:

Lemma 1.2. Let $M$ be a complete noncompact manifold without boundary, such that $M$ supports no nonconstant bounded harmonic functions. Let $g_{1}, \ldots, g_{k}$ be a finite set of bounded superharmonic functions such that $\inf _{M} g_{j}=0$ for all $1 \leq j \leq$ $k$. Let $g=\max \left\{g_{1}, \ldots, g_{k}\right\}$; then $\inf _{M} g=0$.

Lemma 1.2 is basically due to Kendall [Ke], but the method is different. In Lemma 1.2, the assumption is weaker in the sense that there may be bounded harmonic functions defined at the infinity of $M$ which is not asymptotically constant at infinity. However, we have the following: 
Proposition 1.3. Let $M$ be a complete noncompact manifold without boundary, and let $D$ be a bounded domain of $M \backslash \bar{D}$. Suppose there are at least two nonparabolic ends of $M$ with respect to $D$. Let $E$ be one of the nonparabolic ends. Then every bounded harmonic function on $M$ is asymptotically constant at the infinity of $E$ if and only if every bounded harmonic function defined near the infinity of $E$ is asymptotically constant at the infinity of $E$.

Proof. Suppose every bounded harmonic function on $M$ is asymptotically constant at infinity of $E$. Since $M$ has at least two nonparabolic ends, by [L-T 4], there is a nonconstant bounded harmonic function $f$ on $M$ such that $0<f<1$ and

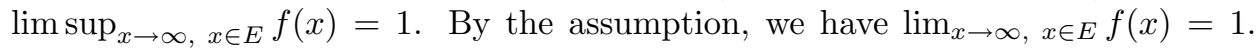
Hence $1-f$ is a positive harmonic function on $M$ such that $(1-f) \rightarrow 0$ at the infinity of $E$. Let $g$ be a harmonic function defined near the infinity of $E$. We can extend $g$ to be a bounded function which is harmonic outside a compact set. For $R>0$, let $h_{R}$ be the harmonic function defined on $B_{o}(R)$, such that $h_{R}=g$ on $\partial B_{o}(R)$, where $o$ is a fixed point. There exists $R_{i} \rightarrow \infty$ such that $h_{R_{i}}$ converges to a bounded harmonic function $h$ on $M$. Since $h_{R_{i}}$ are uniformly bounded on compact sets, $g-h_{R_{i}}$ is harmonic in $B_{o}\left(R_{i}\right) \backslash B_{o}\left(R_{0}\right)$ for some $R_{0}$, and $g-h_{R_{i}}=0$ on $\partial B_{o}(R)$, hence there is a constant $C$ independent of $i$ such that $C(1-f) \geq\left|g-h_{R_{i}}\right|$ for $i$ large enough. Hence $C(1-f) \geq|g-h|$. Since $(1-f) \rightarrow 0$ at the infinity of $E$ and $h$ is asymptotically constant at the infinity of $E$, so $g$ is also asymptotically constant at the infinity of $E$. The converse is obvious.

Using the above results, we generalize Liouville theorems on harmonic maps of Cheng $[\mathrm{Cg}]$, Choi $[\mathrm{Ch}]$ and Kendall $[\mathrm{Ke}]$. Let us first introduce the following definition.

Definition 1.3. Let $N$ be a complete manifold with sectional curvature bounded from above by $k^{2} \geq 0$. Let $B_{p}(R)$ be a geodesic ball in $N$ of radius $R$ and with center $p$, and denote by $C(p)$ the cut locus of its center. We call the ball $B_{p}(R)$ a regular ball in $N$ if $k R<\pi / 2$ and $C(p) \cap B_{p}(R)$ is empty.

Clearly, if $N$ is simply connected with nonpositive sectional curvature, then each geodesic ball in $N$ is regular by the Hadamard's theorem.

Theorem 1.4. Let $M$ be a complete noncompact manifold and $N$ be a complete manifold with sectional curvature bounded above by $k^{2}>0$.

(a) Suppose $M$ has no nonconstant bounded harmonic function; then every harmonic map from $M$ into a regular ball of $N$ must be constant.

(b) Let $D$ be a bounded domain in $M$. Suppose $E$ is an end of $M$ such that every bounded harmonic function defined near the infinity of $E$ is asymptotically constant. Then every harmonic map from $E$ into a regular geodesic ball of $N$ is asymptotically constant at infinity of $E$.

Proof. (a) was proved in [Ke] by using Lemma 1.2. To prove (b), let $u$ be a harmonic map from $E$ into a regular geodesic ball $B_{p}(R)$ of $N$. Let $K=\overline{u(E)}$. Then by [J-K, A-C-M, Ke], for all $q \in K$ the function

$$
\Psi_{q}(s)=\frac{1-\cos \left(k d_{N}(q, s)\right)}{k \cos \left(k d_{N}(p, s)\right)}
$$

is convex in $B_{p}(R)$. Therefore $\Psi_{q}(u(x))$ is a bounded subharmonic function in $E$ by [Go]. Let $c_{q}=\lim \sup _{x \rightarrow \infty, x \in E} \Psi_{q}(u(x))$ and let $g_{q}(x)=c_{q}-\Psi_{q}(u(x))$. So $g_{q}$ 
is bounded and superharmonic, and $\liminf _{x \rightarrow \infty, x \in E} g_{q}(x)=0$. By Lemma 1.1, it is easy to see that for all $\epsilon>0$ and for any finitely many points $q_{1}, \ldots, q_{k}$,

$$
\bigcap_{i=1}^{k}\left\{x \in E \mid \quad g_{q_{i}}(x) \leq \epsilon\right\} \neq \emptyset .
$$

Consider the family $\mathcal{F}$ of subsets $A(p, \epsilon)$ of $K$, for $q \in K$ and $\epsilon>0$, where

$$
A(q, \epsilon)=\left\{s \in K \mid \quad c_{q}-\Psi_{q}(s) \leq \epsilon\right\} .
$$

By (1.6) and the definitions of $K$ and $g_{q}$, we see that the intersection of finitely many $A(p, \epsilon)$ is nonempty. By the compactness of $K$, there is $q_{0} \in A(q, \epsilon)$ for all $q \in K$ and for all $\epsilon>0$. In particular, we have $c_{q_{0}}-\Psi_{q_{0}}\left(q_{0}\right) \leq 0$. Hence $c_{q_{0}} \leq 0$. But $c_{q_{0}}$ is nonnegative, so $c_{q_{0}}=0$. By the definition of $c_{q_{0}}$, we see that $\lim \sup _{x \in E, x \rightarrow \infty} \Psi_{q_{0}}(u(x))=0$. Hence $\lim _{x \in E, x \rightarrow \infty} u(x)=q_{0}$. This completes the proof of the theorem.

By Proposition 1.3, we have the following:

Corollary 1.5. Let $M$ be a complete noncompact manifold and let $D$ be a bounded domain in $M$. Suppose any bounded harmonic function on $M$ is asymptotically constant at each nonparabolic end. Then any harmonic map from $M$ into a regular geodesic ball of a manifold $N$ is also asymptotically constant at each nonparabolic end.

Note that if $M$ has only one nonparabolic end, and $M$ satisfies the condition of the corollary, then $M$ has no nonconstant bounded harmonic function. See [L-T 2].

The method of proof in Theorem 1.4 can be applied to harmonic maps into a complete manifold with nonnegative sectional curvature. Let $N$ be a complete noncompact manifold with nonnegative sectional curvature. Let $p \in N$, and let $\gamma$ be a ray emanating from $p$ parametrized by arclength. Let $\beta_{\gamma}$ be the Busemann function of $\gamma$. That is, $\beta_{\gamma}(x)=\lim _{t \rightarrow \infty}(t-d(x, \gamma(t)))$. Let $\beta_{p}=\sup _{\gamma} \beta_{\gamma}$, where the supremum is taken on the set of all rays $\gamma$ from $p$. It is well known that $\beta_{p}$ is a convex function which is Lipschitz with Lipschitz constant equal to 1 ; see [C-G]. Moreover, $\beta_{p}(p)=0$.

Proposition 1.6. Let $M$ be a complete noncompact manifold and let $D$ be a bounded domain. Assume that every bounded harmonic function defined on an end with respect to $D$ is asymptotically constant. Let $u$ be a bounded harmonic map from $M$ into a complete noncompact manifold with nonnegative sectional curvature. Then for each end $E$ there is a point $p \in N$ such that $\lim _{\sup _{x \rightarrow \infty}, x \in E} \beta_{p}(u(x))=0$.

Proof. The proof is similar to the proof of Theorem 1.4, by considering $\beta_{p} \circ u$. We omit the details.

Examples. (1) Let $M$ be a complete noncompact manifold with asymptotically nonnegative sectional curvature. Namely, there is a monotonic nonincreasing continuous function $k:[0, \infty) \rightarrow[0, \infty)$, such that $\int_{0}^{\infty} t k(t) d t<\infty$, and the sectional curvature of $M$ at $x$ satisfies $K_{M}(x) \geq-k(r(x))$ for all $x$, where $r(x)$ is the distance of $x$ from a fixed point $o$. Then $M$ has finitely many ends and each end satisfies the condition in Theorem 1.4 (b). See [L-T 1] and [K 1, K 2]. This is also true for $\tilde{M}$ which is quasi-isometric to $M$. See $[\mathrm{S}]$. 
(2) Let $M$ be a complete noncompact manifold which is quasi-isometric to a complete manifold with nonnegative Ricci curvature outside a compact set; then $M$ has finitely many ends. If in addition, $M$ has finite first Betti number, then each end satisfies the condition in Theorem 1.4 (b). See [L-T 5], [Cai], and [Liu]. We may relax the condition on the Ricci curvature, provided the manifold satisfies certain conditions on volume comparison. If the condition on the first Betti number is removed, then in some cases, $M$ still satisfies the condition in Proposition 1.3. See [L-T 5] for more details.

In this section, we will prove an existence theorem on harmonic maps and will give more information on bounded harmonic maps considered in $\S 1$.

Theorem 2.1. Let $E_{1}, \ldots, E_{l}$ be the nonparabolic ends of $M$ with respect to some compact set. Suppose that every bounded harmonic function on $M$ is asymptotically constant at infinity of each $E_{i}$. Let $B_{p}(R)$ be a regular geodesic ball in a manifold $N$. Then for any $p_{1}, \ldots, p_{l}$ in $B_{p}(R)$ there exists a harmonic map $u: M \rightarrow N$ with finite total energy such that $u(M) \subset B_{p}(R)$ and $\lim _{x \rightarrow \infty, x \in E_{i}} u(x)=p_{i}$ for all $i=1, \ldots, l$.

Proof. The theorem can be proved as in $[\mathrm{A}-\mathrm{C}-\mathrm{M}]$ using the result of $[\mathrm{H}-\mathrm{K}-\mathrm{W}]$. To be more precise, let $n$ be the dimension of $N$ and let $\left(y_{1}, \cdots, y_{n}\right)$ be the normal coordinates of $B_{p}(R)$ with center at $p$. In this coordinate system, $p_{i}=\left(a_{1 i}, \cdots, a_{n i}\right)$, $1 \leq i \leq l$. As in the proof of Proposition 1.3, by [L-T 4], and using the assumptions, for each $i$ there is a bounded harmonic function $f_{i}$ such that $\lim _{x \rightarrow \infty, x \in E_{i}} f_{i}=1$ and $\lim _{x \rightarrow \infty, x \in E_{j}} f_{i}=0$ for $j \neq i$. Let $h=\left(h_{1}, \cdots, h_{n}\right)$ be the vector-valued harmonic function with $h_{k}=\sum_{i=1}^{l} a_{k i} f_{i}$, and let $v$ be the harmonic function such that $v=\sum_{i=1}^{l}\left(\sum_{k=1}^{n}\left(a_{k i}\right)^{2}\right) f_{i}$. As in [A-C-M], one can find a harmonic map $u$ from $M$ into $B_{p}(R)$ and a constant $C$ such that

$$
d_{N}(u, h) \leq C\left(v-\sum_{k=1}^{n}\left(h_{k}\right)^{2}\right)
$$

By the definition of $h$, it is easy to see that $\lim _{x \rightarrow \infty, x \in E_{i}} u(x)=p_{i}$ for all $i=$ $1, \ldots, l$. Moreover, $h$ has finite Dirichlet integral and hence has finite total energy; see for example [L-T 5]. By the construction of $u$, it is easy to see $u$ has finite total energy.

We also have the following uniqueness result.

Proposition 2.2. Let $u, v$ be two harmonic maps from $M$ into $N$ with sectional curvature bounded from above by $k^{2}>0$, such that their images are contained in a regular ball $B_{p}(R)$ of $N$. Suppose that on each nonparabolic end $E$ of $M$,

$$
\lim _{x \rightarrow \infty, x \in E} u(x)=\lim _{x \rightarrow \infty, x \in E} v(x)
$$

Then $u \equiv v$ on $M$.

Proof. According to $[\mathrm{Ke}]$ there exists a continuous nonnegative bounded convex function $\Psi$ defined on $B_{o}(R) \times B_{o}(R)$ and $\Psi(x, y)=0$ if and only if $x=y$. Consider 
$f(x)=\Psi(u(x), v(x))$. Since both $u$ and $v$ are harmonic maps and $\Psi$ is convex, we conclude $\Delta f \geq 0$ on $M$. Clearly, $f$ is bounded. Hence, $f$ attains its maximum at the infinity of $M$, that is, at the infinity of some end. But the maximum cannot be attained at the infinity of a parabolic end $e$ unless it is a constant. Otherwise $\sup _{M} f-f$ will be a positive superharmonic function on $e$ attaining its infimum at the infinity of $e$, which implies that the end $e$ is nonparabolic. At the infinity of each nonparabolic end, from our assumption, $d_{N}(u(x), v(x)) \rightarrow 0$ as $x \rightarrow \infty$. Thus, $\max _{M} f=0$ and $f \equiv 0$ as $f \geq 0$. Therefore, $u \equiv v$.

Corollary 2.3. Let $M$ be a complete manifold, and let $D$ be a bounded domain in $M$. Assume every bounded harmonic function on $M$ is asymptotically constant near infinity of each nonparabolic end. Then every bounded harmonic map from $M$ into a regular geodesic ball of another manifold $N$ has finite total energy.

Proof. The corollary follows from Corollary 1.5, Theorem 2.1 and Proposition 2.2.

\section{REFERENCES}

[A-C-M] P. Avilés, H.I. Choi, and M. Micallef, Boundary behavior of harmonic maps on nonsmooth domains and complete negatively curved manifolds, J. Functional Anal. 99 (1991), 293-331. MR 92j:58025

[Cai] M. Cai, Ends of Riemannian manifolds with nonnegative Ricci curvature outside a compact set, Bull. AMS 24 (1991), 371-377. MR 92f:53045

[C-G 2] J. Cheeger and D. Gromoll, On the structure of complete manifolds of nonnegative curvature, Ann. of Math. 92 (1972), 413-443. MR 46:8121

[Cg] S. Y. Cheng, Liouville Theorem for Harmonic Maps, Proc. of Symposia in Pure Math. 36 (1980), 147-151. MR 81i:58021

[C-Y] S. Y. Cheng and S. T. Yau, Differential equations on Riemannian manifolds and their geometric applications, Comm. Pure Appl. Math. 28 (1975), 333-354. MR 52:6608

[Ch] H. I. Choi,, On the Liouville theorem for harmonic maps, Proc. AMS 85 (1982), 91-94. MR 83j:53073

[Go] W. B. Gordon, Convex functions and harmonic maps, Proc. AMS 33 (1972), 433-437. MR 45:1075

[G-W] R. E. Green and H. Wu, Function theory on Manifolds which possess a pole, Lecture Notes in Math. 699 (1979), Springer-Verlag.

[H-K-W] S. Hildebrandt, H. Kaul and K. -O. Widman, An existence theory for harmonic mappings of Riemannian manifolds, Acta Math. 138 (1977), 1-16. MR 55:6478

[J-K] Jäger, W. and Kaul, H., Uniqueness and stability of harmonic maps and their Jocobi fields, Manu. Math. 28 (1979), 269-291. MR 80j:58030

[K 1] A. Kasue, A compactification of a manifold with asymptotically nonnegative curvature, Ann. Scient. Ec. Norm. Sup. 21 (1988), 593-622. MR 90d:53049

[K 2] A. Kasue, Harmonic functions with growth conditions on a manifold of asymptotically nonnegative curvature I, Geometry and Analysis on Manifolds (1988), Springer-Verlag Lecture Notes in Mathematics 1339, 158-181. MR 89i:53030

[Ke] W. S. Kendall,, Probability, convexity, and harmonic maps with small image I: uniqueness and fine existence, Proc. London Math. Soc. 61 (3) (1990), 371-406. MR 91g:58062

[L-T 1] P. Li and L. F. Tam, Positive harmonic functions on complete manifolds with nonnegative curvature outside a compact set., Annals Math. 125 (1987), 171-207. MR 88m:58039

[L-T 2] P. Li and L. F. Tam, Symmetric Green's functions on complete manifolds, Amer. J. Math. 109 (1987), 1129-1154. MR 89f:58129

[L-T 3] P. Li and L. F. Tam, Complete surfaces with finite total curvature, J. Diff. Geom. 33 (1991), 139-168. MR 92e:53051

[L-T 4] P. Li and L. F. Tam, Harmonic functions and the structure of complete manifolds, J. Diff. Geom. 35 (1992), 359-383. MR 93b:53033 
[L-T 5] P. Li and L. F. Tam, Green's Functions, Harmonic Functions and Volume Comparison, J. Diff. Geom. 41 (1995), 277-318.

[Liu] Z-D. Liu, Ball covering on manifolds with nonnegative Ricci curvature near infinity, preprint.

[S] C. J. Sung, Harmonic functions under quasi-isometry, to appear in J. Geom. Anal..

[Y] S. T. Yau, Harmonic functions on complete Riemannian manifolds, Comm. Pure Appl. Math. 28 (1975), 201-228. MR 55:4042

Department of Mathematics, National Chung Cheng University, Chia-Yi, Taiwan 62117

E-mail address: cjsung@math.ccu.edu.tw

Department of Mathematics, The Chinese University of Hong Kong, Shatin, Hong KONG

E-mail address: lftam@math.cuhk.hk

Department of Mathematics, Stanford University, Stanford, California 94305

E-mail address: jwang@math.stanford.edu 